\title{
Role of Intratympanic Methylprednisolone in Sudden Sensorineural Hearing Loss. A Clinical Study
}

\author{
Dr. Preeti Sharma ${ }^{1}$, Dr Saurav Shekhar ${ }^{2}$, Dr. Satish Kumar ${ }^{3}$, \\ Dr. P N P Pal ${ }^{4}$, Dr. B B Bajpayi ${ }^{5}$ \\ ${ }^{1,3,4,5}$ Department Of ENT, Patna Medical College Hospital, Patna \\ ${ }^{2}$ Department of Anesthesiology, Indira Gandhi Institute Of Medical Sciences, Patna
}

\begin{abstract}
Objective: Sudden sensorineural hearing loss (SSNHL) is a common emergency in Otology. The etiology, clinical features and treatment remains a matter of debate for over decade. The aim of this study is to evaluate the effectiveness of intratympanic steroid therapy (IST) in patients with idiopathic sudden sensorineural hearing loss (ISSHL), determining the overall hearing recovery and comparing the results with different variables.

Materials and Methods: Our study consisted of 50 patients with sudden sensorineural hearing loss. The patients received $0.5 \mathrm{ml}$ +of methylprednisolone by direct intratympanic injection. Results showed overall PTA improvement in 42 patients (84\%), 08 (16\%) had no change in hearing. There was a significant statistical correlation between hearing recovery and time to onset of symptoms, severity of hearing loss and frequency of hearing loss.
\end{abstract}

Conclusions: IST is an effective and safe therapy in sudden sensorineural hearing loss cases. Early intratympanic injection, hearing losses less than $90 \mathrm{~dB}$ and the involvement of the low frequencies seem to influence the hearing recovery positively.

Keywords: Sensorineural Hearing loss, Intratympanic Steroid Therapy, Methylprednisolone

\section{Introduction}

Sudden sensorineural hearing loss (SSNHL) is a common emergency in Otology and is defined as a decline in hearing over 3 days or less affecting 3 or more frequencies by $30 \mathrm{~dB}$ or greater with no identifiable etiology ${ }^{1,2}$. Sudden SNHL affects between 1 person per 10,000 year or approximately 4,000 new cases annually in the United States. The hearing loss is nearly always unilateral and is commonly associated with tinnitus $(>90 \%)$, vertigo and aural fullness. The true incidence of sudden SNHL is probably underestimated because many who recover hearing early (within the first few days) are unlikely to seek medical advice.

The etiology, natural history, and treatment of this disorder have been subjects of debate for many years. The actual number of patients recovering spontaneously from sudden SNHL without having sought medical attention is unknown. The high rate of spontaneous recovery (up to 65\%), also confounds reviews as to the therapeutic efficacy of any single agent or therapeutic interventions ${ }^{3}$. Otologists are still searching answers to the etiology, physiopathology and therapeutical management of this disorder. The most common theories of the etiology of idiopathic SSHL include viral infection of the cochlear or labyrinth nerve, vascular occlusion with microcirculatory disturbances, immunologic diseases, and intra labyrinthine membrane breaks ${ }^{4}$. Many treatments for ISSHL have been tested and found ineffective. These include hyperbaric oxygen, agents that decrease blood viscosity (osmotic diuretics, pentoxifylline, pro-caine, and heparin), vasodilator drugs (histamine, papaverine, verapamil and carbogen), free radical scavenging vitamins, steroids and magnesium. Many factors seem to influence recovery. Degree of hearing loss, audiogram pattern, existence of vertigo, and duration between the onset and treatment of SNHL are probably the most important factors.

There are several theoretical advantage of intratympanic steroid over gastrointestinal or intravenous steroid delivery ${ }^{2}$. It produces significantly higher perilymph concentrations of steroids administered and it is possible to reduce the side effects due to systemic absorption. The intratympanic steroids treatment (IST) procedure is well tolerated and an office-based procedure done under local (topical) anesthesia. Unlike systemic therapies, intratympanic therapy allows for the selection of the affected ear to be treated. In addition to glucose intolerance and avascular necrosis of the hip, other less severe side effects of systemic steroids such as insomnia, irritability, gastritis, and mood changes may potentially be avoided with topical therapy. The primary disadvantage of intratympanic steroids is the lack of proven efficacy and/or superiority over systemic steroids. Other potential disadvantages include pain, tympanic membrane perforation, acute otitis media, otorrhea, vertigo, and the potential for further hearing loss.

The aim of this study is to study for the safety and effectiveness of IST in the treatment of ISSHL, with special attention for the correlations between hearing recovery and time to onset of therapy, severity and frequencial range of hearing loss, age of the patient, and status of the contralateral ear. 


\section{Materials And Methods}

This prospective clinical study was done in 50 patients who had a sudden unilateral hearing loss of at least $30 \mathrm{~dB}$ across 3 contiguous frequencies occurring in less than 72 hours. Patients didn't had any history of otological surgery, Meniere disease, autoimmune / radiation induced or genetic hearing loss, or any other potential etiology for sensorineural hearing loss. There was no evidence of retrocochlear disease evident on magnetic resonance imaging. There was no history of acoustic or barotraumas, no exposure to ototoxic drugs and no history of meningitis. All patients had normal otoscopic examination and had not taken any conventional therapies in two previous weeks.

\section{Audiometric Data}

Patients were evaluated using standardized methods for pure-tone threshold audiometry and speech discrimination score by audiologists pre- and post injection. Pure-tone average (PTA) was calculated as an average of the threshold measured at $0.5,1.0$, and $2.0 \mathrm{KHz}$ Speech discrimination score (SDS) was tested by calculating the percent correct of a phonetically balanced, monosyllabic word list. Auditory measurements were performed before and 1 month after the treatment, according to Siegel's criteria for hearing improvement. 'Complete recovery' was defined as more than $30 \mathrm{~dB}$ hearing gain and as final hearing better than $25 \mathrm{~dB}$. 'Partial recovery' described as more than $15 \mathrm{~dB}$ hearing gain and as final hearing between 25 and $45 \mathrm{~dB}$, 'slight improvement' as more than $15 \mathrm{~dB}$ hearing gain but with a final hearing poorer than $45 \mathrm{~dB}$, and 'no improvement' as less than $15 \mathrm{~dB}$ hearing gain and final hearing poorer than $75 \mathrm{~dB}$

Operative Procedure of Intratympanic Injection: The operative procedure for intratympanic steroid injection was performed under a microscope in a supine patient. External auditory canal was cleaned and after confirmation of intact tympanic membrane and normal middle ear status, local anaesthesia was administered with a cotton ball soaked with $10 \%$ lignocaine, which was applied over tympanic membrane for 20 minutes. With patient's head tilted to $45^{\circ}$ towards healthy side, a 25 -gauge spinal needle was introduced into the posteroinferior portion of tympanic membrane and $0.4-0.5 \mathrm{~mL}$ of methylprednisolone $(40 \mathrm{mg} / \mathrm{mL})$ was administerd slowly so that drug pooled around the round window niche. Patient was instructed to avoid swallowing or moving for 30minutes, remaining in the same position to provide maximal absorption of the drug and to prevent leakage through the Eustachian tube. IST was performed on $1^{\text {st }}, 3^{\text {rd }}$ and $5^{\text {th }}$ day, up to 4 total injections, one every two or three days.

\section{Results}

50 patients were included in the study. There were 22 men (44\%) and 28 women (56\%). The mean age at enrolment for all patients was 46.4 years and ranged from 30 to 75 years. The mean age for the men was 52.7 years and for the women was 44.7 years

Overall Hearing Recovery: Overall, 42 patients (84\%) showed improvement in PTA, 08 (16\%) had no change in hearing. According to Siegel's criteria, 19 patients showed "complete recovery", 16 patients showed "partial recovery" and 7 patients had "slight recovery".

Recovery Related to IST Time to Onset of Symptoms: The average number of days from onset of symptoms to intratympanic therapy was 13 days with a range of 5 days to 30 days. For the group that responded to IST with a "complete recovery" ( $\mathrm{n}=19)$, the mean was 9 days; for the group that responded to IST with a "partial or slight recovery" $(\mathrm{n}=23)$, the mean was 17 days; for the group that did not respond $(\mathrm{n}=08)$, the mean was 25 days. Statistical analysis shows that there is a significant correlation between hearing recovery and IST time to onset of symptoms.

Recovery Related to Severity of Hearing Loss: Total of 15 patients (30\%) had hearing loss greater than $90 \mathrm{~dB}$ with an improvement rate of $8.1 \%$; 26 patients $(52 \%)$ having hearing loss of $90 \mathrm{~dB}-50 \mathrm{~dB}$ had improvement rate of 24\%; 9 patients $(18 \%)$ had hearing loss in the range of $50 \mathrm{~dB}$ - 30dB showed an improvement rate of $54 \%$. Patients with severe hearing loss greater than $90 \mathrm{~dB}$ had poorer recovery compared with those having hearing loss less than $90 \mathrm{~dB}$.

Recovery Related to Frequency of Hearing Loss: We have studied the hearing recovery for each frequency $(0.5,1,2,4$, and $8 \mathrm{kHz})$ of hearing threshold. A total of 35 patients( $70 \%)$ showed improvement over $30 \mathrm{~dB}$ on hearing gain for the frequency of 0.5 The same result was obtained with $1 \mathrm{kHz}$ frequency in 26 patients $(52 \%)$. with $2 \mathrm{kHz}$ frequency in 21 patients (42\%), with $4 \mathrm{kHz}$ frequency in 13 patients (26\%), and with $8 \mathrm{kHz}$ frequency in 8 patients $(16 \%)$. Statistical analysis shows a significant correlation between recovery and low frequencies $(0.5$ and $1 \mathrm{kHz})$ of hearing threshold.

Recovery Related to Status of the Contra lateral Ear: $76.5 \%$ patients had normal hearing in the contra lateral ear. The recovery rate in this group was $31.5 \%$. Only $23.5 \%$ of patients had abnormal hearing in 
the opposite ear. The recovery rate in this group was $27.5 \%$. Statistical analysis showed no significant correlations between recovery and situation of the contralateral ear
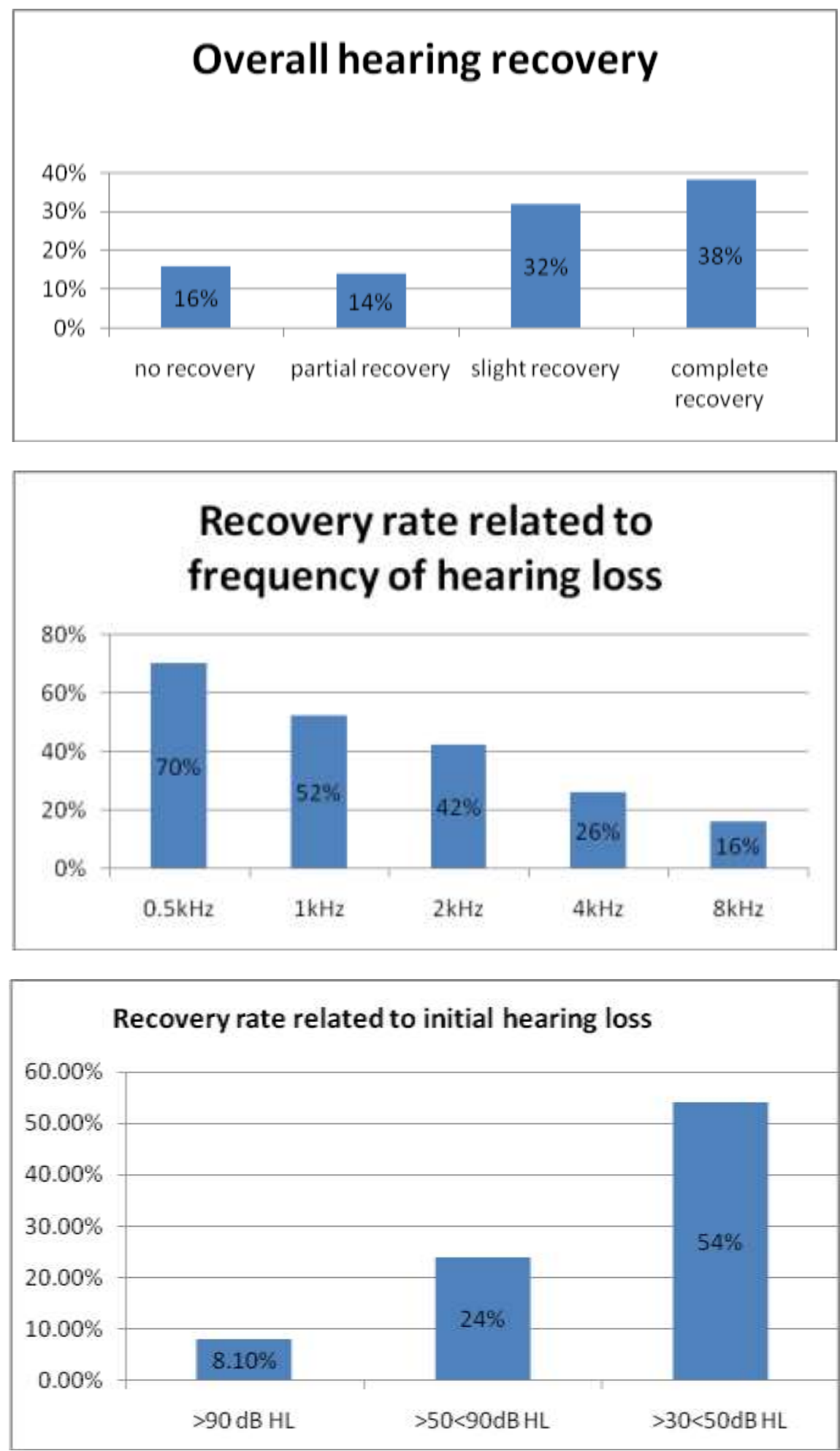

\section{Discussion}

Sudden sensorineural hearing loss (SSNHL), a common otologic emergency, has a tendency to show spontaneous hearing improvement. All reports describing treatments indicate that early initiation of treatment will undoubtedly lead to improved prognosis. The definite mechanism through which steroids may improve hearing is still not very clear. Both glucocorticoid and mineralocorticoid receptors may be found in the inner ear. The main roles of steroids in the treatment of SSNHL are: ${ }^{5,6}$ 
- Protection of cochlea from the harmful effects of inflammatory mediators, such as the tumor necrosis factor (TNF- $\alpha$ and NF- $\mathrm{kB}$ ) and cytokines (interleukin 1 and 6), which is elevated in infection and inflammation

- Increasing cochlear blood flow thereby avoiding cochlear ischemia;

- Avoiding noise-induced hearing loss;

- Regulating protein synthesis in the inner ear.

Vascular stria which regulates secretion of $\mathrm{Na} / \mathrm{K}$ in order to maintain endocochlear potential is the most frequent site of injury in the SSNHL.Systemic steroid therapy improves vascular stria function and may preserve its morphology and therefore it has potential for recovering from SSNHL.The first randomized controlled trial of SSNHL therapy was performed by Wilson et al. ${ }^{7}$ They investigated therapeutic efficacy in a study of 67 patients with SSNHL who were given either steroid or a placebo. The results showed that overall $61 \%$ of the steroid group showed a hearing improvement, compared to placebo group and there was a strong correlation between the pretreatment audiogram and the results. Patients with thresholds of $40 \mathrm{~dB}$ or less or with mid-frequency losses of up to $85 \mathrm{~dB}$ invariably had excellent hearing recovery. Silverstein ${ }^{8}$ in 1996 first reported intratympanic steroid in treatment of sudden SNHL followed by Parnes ${ }^{9}$ in 1999 and thereafter several reports have been published regarding this therapy mode. Silverstein ${ }^{8}$ reviewed 46 patients treated with transtympanic steroids for a variety of disorders, of which 8 patients had diagnosis of sudden SNHL. One patient showed improvement in speech reception thresholds from $110 \mathrm{~dB}$ to $85 \mathrm{~dB}$ and another from $75 \%$ to $65 \%$ SRT. ${ }^{12}$ Parnes et al. ${ }^{9}$ treated 37 patients presenting within 6 weeks of sudden SNHL ${ }^{13}$ with intratympanic methylprednisolone (9 patients) and intratympanic dexamethasone (4 patients). Six patients showed significant improvement in hearing thresholds, with 5 progressing from a severe or profound loss to relatively normal thresholds. No correlation between outcome and time of treatment after HL was noted.13 Chandrasekhar $^{10}$ treated 10 patients with dexamethasone intratympanically. The time interval between onset of hearing loss and treatment averaged 33 days. Overall improvement was noted with a mean improvement of 9-dB PTA and 15.8\% discrimination. Improvement was noted in all patients with diabetes and Meniere disease. Patients with long intervals to treatment, downsloping audiogram, and surgical trauma to the inner ear did not show recovery with intratympanic steroids.

Lefebvre and Staecker $^{11}$ treated 6 patients having failed medical therapy with methylprednisolone infusion through a microcatheter for 8 to 10 days. All patients showed improvement in hearing thresholds with an average of 16.25 to $25 \mathrm{~dB}$ improvement in thresholds. Gianoli and $\mathrm{Li}^{2}$ performed prospective study on patients treated with intratympanic steroids (dexamethasone or methylprednisolone). A change of greater than or equal to $10 \mathrm{~dB}$ in the PTA or speech reception threshold or $10 \%$ in speech discrimination was considered a positive response. A $44 \%$ response rate was noted in these prior treatment failures with the average improvement of $15.2 \mathrm{~dB}$ and $21 \%$ SDS. Ho et al. ${ }^{12}$ studied 39 patients with sudden SNHL in which $29(74 \%)$ failed systemic steroids and were randomized into 2 treatment groups. Fifteen patients received intratympanic steroid therapy and 14 were continued on further medical therapy (without steroids). They noted 53\% improvement in the intratympanic steroid group as opposed to $7.1 \%$ for the noninjected group using 30-dB gain in PTA as criteria for successful outcome.

Herr and Marzo $^{13}$ reported on 17 patients treated with transtympanic steroids through a Microwick and/or round window catheter placement. All patients had failed prior systemic therapy with prednisone and were treated from 2 to 20 weeks after onset of HL. Overall, 53\% showed improvement in thresholds after treatment with average improvement being $24.3 \mathrm{~dB}$. Battista ${ }^{14}$ enrolled 25 patients with profound SNHL in whom both systemic and intratympanic steroids were used concomitantly. Overall poor results were achieved in this population of profound HL patients with only $12 \%$ (3 of 25) achieving a full or partial response. Slattery et al. reported 20 patients treated with methylprednisolone for sudden SNHL that failed systemic steroids. Fifty-five percent showed clinically significant (10-dB PTA or 12\% discrimination) improvement in hearing and tinnitus. In 2006, Dallan et al. ${ }^{15}$ treated 8 patients with intratympanic methylprednisolone in a prospective study, with $75 \%$ improving after a single injection. Choung et al. ${ }^{16}$ had a $38 \%$ improvement following with intratympanic therapy compared to $6.1 \%$ improvement in a control group treated with systemic therapy alone. Xenellis ${ }^{17}$ showed a $47 \%$ improvement in patients with intratympanic therapy following treatment failure, while none of the patients in a matched control improved over time.

With the natural history of sudden SNHL suggesting a high recovery rate, it is difficult to determine if any therapeutic intervention actually improves hearing recovery. The natural history of untreated patients with sudden SNHL ranges from recovery rates of $31 \%$ to $65 \%$. The range of hearing recovery reported in the literature in treated patients ranges from $35 \%$ to $89 \%$.Several reasons may explain the significant differences in reported recovery rates between studies; however, the best explanation may lie in what is considered a "successful" treatment. 
According to our study IST appear to be more effective on the low frequency hearing loss. Since the intratympanic steroid spreads into the perilymph through the round window, it would be actually expected that hearing improvement might occur in high frequencies (basal turn of the cochlea) than in low frequencies (apex of the cochlea). The differential vulnerability of basal and apical hair cells seems to explain this clinical result. The basal turn of the cochlea is more vulnerable to trauma and free radicals than the apical turn; in daily clinical practice, the hearing loss from noise, ototoxic drugs, or trauma easily occurs in the high frequencial range involving the cochlear base. Besides, the outer and inner hair cells of the cochlear base develop ultrastructural anomalies more quickly than those in the apical turns following severe or total cochlear ischemia.

\section{Conclusion}

Our study suggested that intratympanic steroid therapy is effective and safe treatment of SSNHL and moreover, hearing losses less than $90 \mathrm{~dB}$, involvement of the low frequencies and early starting of IST seem to positively influence the hearing recovery. Our study though had limitations such as small sample size and the results may be affected by many factors such as the natural course of recovery of SSNHL, patient age and severity of deafness. Many more multi-centric well-controlled clinical trials are warranted to document the real efficacy of intratympanic steroid injection in the treatment of idiopathic SSNHL.

\section{References}

[1]. Haberkamp TJ, Tanyeri HM. Management of idiopathic sudden sensorineural hearing loss. Am J Otol 1999;20:587-95

[2]. Gianoli GJ, Li JC. Transtympanic steroid for treatment of sudden hearing loss. Otolaryngol Head Neck Surg 2001;125:142-6.

[3]. Mattox DE, Simmons FB. Natural history of sudden sensori-neural hearing loss.Ann Otol Rhinol Laryngol1977;86:

[4]. Merchant SN, Adams JC, Nadol JB. Pathology and pathophysiology of idiopathic sudden sensorineural hearing loss. Otol Neurotol 2005;26:151-60.

[5]. Rarey KE, Luttge WG. Presence of type I and type II/IB receptors for adrenocorticosteroid hormones in the inner ear. Hear Res 1989;41:217-22.

[6]. Gloddek B, Lamm K, Arnold W. Pharmacological influence on inner ear endothelial cells in relation to the pathogenesis of sensorineural hearing loss. Adv Otorhinolaryngol 2002;59:75-83

[7]. Wilson WR, Byl FM, Laird N. The efficacy of steroids in the treatment of idiopathic sudden hearing loss. A double-blind clinical study. Arch Otolaryngol1980;1463-480.06:772-776.

[8]. Silverstein H, Choo D, Rosenberg SI, Kuhn J, Seidman M,Stein I. Intratympanic steroid treatment of inner ear dis-ease and tinnitus (preliminary report).Ear Nose Throat J 1996;75:468-471.

[9]. Parnes LS, Sun AH, Freeman DJ. Corticosteroid pharmaco-kinetics in the inner ear fluids: an animal study followed by clinical application.Laryngoscope1999;109:1-17

[10]. Chandrasekhar SS. Intratympanic dexamethasone for sudden sensorineural hearing loss: clinical and laboratory evaluation. Otol Neurotol 2001;22:18-23.

[11]. Lefebvre PP, Staecker H. Steroid perfusion of the inner ear for sudden sensorineural hearing loss after failure of conventional therapy: a pilot study. Acta Otolaryngol 2002; 122:698-702

[12]. Ho GM, Lin HG, Shu MT. Effectiveness of intratympanic dexamethasone injection in sudden deafness patients as salvage treatment. Laryngoscope 2004;114:1184-1189.

[13]. Herr BD, Marzo SJ. Intratympanic steroid perfusion for refractory sudden sensorineural hearing loss. Otolaryngol Head Neck Surg 2005; 132:527-531

[14]. Battista RA. Intratympanic dexamethasone for profound idiopathic sudden sensorineural hearing loss. Otolaryngol Head Neck Surg 2005;132:902-905.

[15]. Dallan I, Bruschini P, Nacci A, et al. Transtympanic steroids as a salvage therapy in sudden hearing loss: preliminary results. ORL J Otorhinolaryngol Relat Spec 2006;68: 247 -252.

[16]. Choung YH, Park K, Shin YR, Cho MJ. Intratympanic dexamethasone injection for refractory sudden sensorineural hearing loss. Laryngoscope 2006;116:747-752.

[17]. Xenellis J, Papadimitriou N, Nikolopoulos T, et al. Intratympanic steroid treatment in idiopathic sudden sensorineural hearing loss: a control study. Otolaryngol Head Neck Surg 2006;134:940-945 